\title{
Genetic Analysis of Earliness and Yield Component Traits in Five Barley Crosses
}

\author{
M. Mansour \\ Barley Research Dept., Field Crops Research Institute, ARC, Egypt
}

\begin{abstract}
7 HE PRESENT investigation was carried out at Sakha Agricultural Research Station during 2013/014 to 2015/016 seasons to estimate genetic variance components and type of gene action controlling earliness and yield components of five barley crosses. These crosses used six-population model to determine the intra - and inter-allelic gene interactions controlling the inheritance of earliness and yield traits in five crosses of barley. Mean effect was highly significant for all traits in all crosses. Dominance $\times$ dominance was greater in magnitude than other components in most studied traits, indicating that these traits were greatly affected by dominance and its non-allelic interactions. Significant and highly significant desirable percentages of heterosis relative to mid and better parent were exhibited for grain filling period in crosses no. 1, 3 and 5, plant height in crosses no. 1 and 5, no. of grains/spike in crosses no. 1 and 4, no. of spikes/plant in crosses no. 2, 3 and 4 and grain yield/plant in cross no. 4. Broadsense heritability values were high in all crosses for all studied traits. Narrow-sense heritability and genetic advance ranged from low to medium in most cases due to the opposite directions of dominance and dominance $\mathrm{x}$ dominance effects. Generally, the most promising crosses were the two crosses 1 and 5 for earliness and crosses no. 2, 3 and 4 for grain yield found to be higher in magnitude, which had high genetic advance associated with high heritability and would be of interest in breeding programs for improving barley.
\end{abstract}

Keywords: Barley, Early mature, Six-parameter model, Gene action, Heterosis, Heritability, Genetic advance

\section{Introduction}

Barley (Hordeum vulgare L.) is considered one of the most important cereal crops ranking the fourth in the world cereal crop production. It has a high tolerance to abiotic stresses such as salinity, drought, frost and heat. It is used mainly for animal feeding, brewing malts and human food in some countries. Barley in Egypt planted in the old land after harvesting vegetables grown for exporting has increased. Developing early-maturing barley is important for increasing cultivated area of barley. Early maturing cultivars are also preferable to escape disease, pests, drought, heat and other stress injuries that occur at the end of growing season. Breeding early-maturing cultivars is an important objective in barley breeding programs. Information about the inheritance of earlymaturing and its attributes, as well as yield and its components are very scanty to barley breeders in developing short duration cultivars. Successful breeding programs need continuous information about the genetic variation and systems governing earliness attributes, as well as grain yield and its components (Abdel-Nour, 2011). Contradictory results were obtained by several authors with respect to genetic systems governing these characteristics. Reports are conflicting as to the effect of early vs. late heading genotypes on grain filling. Several researchers indicated that the final grain yield was more related to the rate of grain filling (Mou et al., 1994). Meanwhile, Gebeyehou et al. (1982) reported that grain filling duration was more important than the grain filling rate.

Therefore, it has become necessary to develop early- maturing and high-yielding barley genotypes. The present work was undertaken to study the role of different intra and inter-allelic gene interactions, controlling the inheritance of earliness and yield traits in five barley crosses.

\section{Materials and Methods}

The present investigation was carried out at the experimental field of Sakha Agricultural Research 
Stations, Agricultural Research Center (ARC), during three successive seasons from 2013/2014 to 2015/2016. Six barley genotypes representing a wide range of variability in earliness and grain yield traits were used as parents of five crosses in this study (Table 1).

In the first season $(2013 / 2014)$ five crosses; 1 (Giza $126 \times$ Sico), 2 (Giza $126 \times$ Line-1), 3 (Giza $126 \times$ Line-2), 4 (Giza $132 \times$ Line-2) and 5 (Rihane-03 $\times$ Sico) were hand made to obtain their $F_{1}$ seeds. In the second season (2014/2015), the hybrid seeds were sown and $F_{1}$ plants of each cross were backcrossed to their respective parents to produce the two backcrosses ( $\mathrm{BC} 1$ 's and BC2's). At the same time, the $\mathrm{F}_{1}$ plants were selfed to produce $F_{2}$ seeds. In the third season (2015/2016), the obtained seeds of these populations, i.e. $\mathrm{P}_{1}$ 's, $\mathrm{P}_{2}$ 's, $\mathrm{F}_{1}$ 's, $\mathrm{F}_{2}$ 's, $\mathrm{BC}_{1}$ 's and $\mathrm{BC}_{2}$ 's for the five crosses were sown in the field using a randomized complete blocks design (RCBD) with three replications. Rows were $1.5 \mathrm{~m}$ long, $30 \mathrm{~cm}$ width and the space from plant to plant in a row was $10 \mathrm{~cm}$. Each plot consisted of two rows for each $\mathrm{P}_{1}, \mathrm{P}_{2}, \mathrm{~F}_{1}$, ten rows for $\mathrm{F}_{2}$ and three rows for each of $\mathrm{BC}_{1}, \mathrm{BC}_{2}$ in each replicate. All agricultural practices were followed according to the growing barley recommendations. Data were recorded on 30 individual guarded plants in each $\mathrm{P}_{1}, \mathrm{P}_{2}$ and $\mathrm{F}_{1}, 90$ plants in each $\mathrm{BC}_{1}$ and $\mathrm{BC}_{2}$ and 300 plants in each $\mathrm{F}_{2}$ for days to $50 \%$ heading, days to $50 \%$ physiological maturity, grain filling period (days), grain filling rate (g/days), plant height $(\mathrm{cm})$, spike length $(\mathrm{cm})$, number of grains/ spike, number of spikes/plant, 100 kernel weight (g) and grain yield/plant (g). Heterosis (\%) was calculated as the percentage increase of $F_{1}$ over the mid- and better parent values.

\section{Statistical and genetic analysis}

To determine the presence or absence of non- allelic interactions, scaling test as outlined by Mather (1949) was used. Generation mean analysis was performed according to Mather and Jinks (1982). Genetic analysis of generation means to give estimates of the types of gene action was performed using the relationships given by Gamble (1962). Heritability estimates were computed in both broad $\left(\mathrm{H}^{2}\right)$ and narrow $\left(\mathrm{h}^{2}\right)$ senses for $\mathrm{F}_{2}$ generation according to Allard (1960) and Mather (1949). The expected genetic advance from selection $\left(\mathrm{G}_{\mathrm{a}}\right)$ was calculated according the formulae proposed by Johnson et al. (1955), using the selection differential $(\mathrm{k})$ equal 2.06 for $5 \%$ selection intensity and heritability in narrow sense. The predicted genetic advance from selection was expressed as percentage of $\mathrm{F}_{2}$ mean $\left(\mathrm{G}_{\mathrm{a}} \%\right)$ according to Miller et al. (1958).

\section{$\underline{\text { Results and Discussion }}$}

\section{Mean performance}

Means and variances of the studied traits in the six crosses for the six populations $\mathrm{P}_{1}, \mathrm{P}_{2}, \mathrm{~F}_{1}, \mathrm{~F}_{2}$, $\mathrm{BC}_{1}$ and $\mathrm{BC}_{2}$ are presented in Table 2 . The $\mathrm{F}_{1}$ mean values exceeded the mid values of the two parental means for most of studied traits in the six crosses.

The $\mathrm{F}_{2}$ population mean performance values were intermediate between the two parents and less than $F_{1}$ mean performance in most studied traits, indicating the importance of non-additive components of genetic variance for the studied traits. However, the two populations $\left(\mathrm{BC}_{1}\right.$ and $\mathrm{BC}_{2}$ ) mean performance values varied in each trait tended towards the mean of its recurrent parent. The high means of $\mathrm{BC}_{1}$ for days to heading and days to maturity are due to lateness of the first parent $\left(\mathrm{P}_{1}\right)$, while the low means of $\mathrm{BC}_{2}$ are due to the earliness of the second parent $\left(\mathrm{P}_{2}\right)$. The highest magnitude of variance was reported by the $\mathrm{F}_{2}$ generation for all studied earliness and yield traits in all crosses (Table 2) followed by that of backcross generations $\left(\mathrm{BC}^{\prime}{ }_{1} \mathrm{~s}\right.$ and $\left.\mathrm{BC}_{2}{ }_{2} \mathrm{~s}\right)$, while the lowest $\mathrm{S}^{2}$ magnitude was exhibited by $\mathrm{P}_{1}, \mathrm{P}_{2}$ and $F_{1}$ genotypes, which is also logic from the breeding point of view, due to the homogeneity of such genotypes.

TABLE 1. Name and pedigree of the six parental barley genotypes used in this study

\begin{tabular}{lcc}
\hline Pedigree & Genotype & No \\
\hline BaladiBahteem/SD729-por12762-Bc & Giza 126 & 1 \\
Rihane-05//AS46/Athes*2Athe/Lignee686 & Giza 132 & 2 \\
As 46//Avt/Aths & Rihane-03 & 3 \\
Exotic variety & Sico & 4 \\
Cen/Bglo'S'//Kataf-01 & Line-1 & 5 \\
Apm/ HC 1905// Robur/ 3/ Arar/ 4/ Arar/3/ Mari/ Aths *2// M - Att - 73-337 - 1 & Line-2 & 6 \\
\hline
\end{tabular}

Egypt. J. Sus. Agric. Sci . 43, No. 3 (2017) 
TABLE 2. Mean $\left(\mathrm{X}^{-}\right)$and variance $\left(\mathrm{S}^{2}\right)$ of the six populations of the five crosses for days to heading, days to maturity, grain filling period and grain filling rate

\begin{tabular}{|c|c|c|c|c|c|c|c|c|}
\hline Trait & Cross & $\begin{array}{l}\text { Statistical } \\
\text { Parameter }\end{array}$ & P1 & P2 & F1 & F2 & BC1 & BC2 \\
\hline \multirow{8}{*}{$\begin{array}{l}\text { Days to heading } \\
\text { (day) }\end{array}$} & \multirow{2}{*}{1} & $\mathrm{X}^{-}$ & 81.10 & 69.20 & 80.30 & 75.32 & 79.44 & 73.04 \\
\hline & & $\mathrm{S}^{2}$ & 0.69 & 0.58 & 0.42 & 26.47 & 23.25 & 20.71 \\
\hline & \multirow{2}{*}{2} & $\mathrm{X}^{-}$ & 82.01 & 73.20 & 80.90 & 77.70 & 80.16 & 78.12 \\
\hline & & $\mathrm{S}^{2}$ & 0.72 & 0.37 & 0.71 & 17.40 & 14.43 & 13.54 \\
\hline & \multirow{2}{*}{3} & $\mathrm{X}^{-}$ & 81.66 & 65.11 & 76.40 & 76.02 & 79.24 & 74.44 \\
\hline & & $\mathrm{S}^{2}$ & 0.81 & 0.77 & 1.28 & 29.85 & 25.89 & 24.63 \\
\hline & \multirow{2}{*}{4} & $\mathrm{X}^{-}$ & 89.10 & 64.80 & 79.30 & 76.58 & 82.16 & 75.76 \\
\hline & & $\mathrm{S}^{2}$ & 0.88 & 0.79 & 0.42 & 18.06 & 13.35 & 15.83 \\
\hline \multirow{12}{*}{$\begin{array}{l}\text { Days to maturity } \\
\text { (day) }\end{array}$} & \multirow{2}{*}{5} & $\mathrm{X}^{-}$ & 87.10 & 68.94 & 80.40 & 77.93 & 79.84 & 75.36 \\
\hline & & $\mathrm{S}^{2}$ & 0.93 & 0.58 & 1.08 & 15.88 & 12.97 & 11.69 \\
\hline & \multirow{2}{*}{1} & $\mathrm{x}^{-}$ & 120.30 & 103.50 & 113.23 & 107.38 & 108.92 & 103.84 \\
\hline & & $\mathrm{S}^{2}$ & 1.20 & 1.43 & 2.36 & 27.16 & 22.45 & 24.62 \\
\hline & \multirow{2}{*}{2} & $\mathrm{X}^{-}$ & 119.70 & 104.60 & 113.63 & 111.58 & 113.48 & 109.56 \\
\hline & & $\mathrm{S}^{2}$ & 1.25 & 2.21 & 1.59 & 20.74 & 18.52 & 18.22 \\
\hline & \multirow{2}{*}{3} & $\mathrm{X}^{-}$ & 120.07 & 103.01 & 114.00 & 114.23 & 115.16 & 105.20 \\
\hline & & $\mathrm{S}^{2}$ & 1.32 & 1.82 & 1.86 & 29.44 & 22.76 & 27.57 \\
\hline & \multirow{2}{*}{4} & $\mathrm{X}^{-}$ & 122.60 & 102.55 & 116.20 & 113.42 & 115.96 & 109.04 \\
\hline & & $\mathrm{S}^{2}$ & 2.73 & 1.65 & 2.03 & 39.88 & 33.36 & 32.85 \\
\hline & \multirow{2}{*}{5} & $\mathrm{X}^{-}$ & 122.10 & 103.50 & 114.80 & 116.53 & 115.28 & 110.40 \\
\hline & & $\mathrm{S}^{2}$ & 2.58 & 2.25 & 1.20 & 28.14 & 19.42 & 25.62 \\
\hline \multirow{10}{*}{$\begin{array}{l}\text { Grain filling } \\
\text { period } \\
\text { (day) }\end{array}$} & \multirow[b]{2}{*}{1} & $\mathrm{X}^{-}$ & 39.20 & 34.30 & 32.93 & 32.07 & 29.48 & 30.80 \\
\hline & & $\mathrm{S}^{2}$ & 3.25 & 2.28 & 2.27 & 34.58 & 26.25 & 30.97 \\
\hline & \multirow{2}{*}{2} & $\mathrm{X}^{-}$ & 37.69 & 31.40 & 32.73 & 33.88 & 33.32 & 31.44 \\
\hline & & $\mathrm{S}^{2}$ & 3.25 & 3.23 & 2.29 & 45.25 & 38.06 & 34.76 \\
\hline & \multirow{2}{*}{3} & $\mathrm{X}^{-}$ & 38.41 & 37.90 & 37.60 & 38.22 & 35.92 & 30.76 \\
\hline & & $\mathrm{S}^{2}$ & 3.26 & 2.25 & 2.24 & 60.61 & 51.99 & 55.97 \\
\hline & \multirow{2}{*}{4} & $\mathrm{X}^{-}$ & 33.50 & 37.75 & 36.90 & 36.83 & 33.80 & 33.28 \\
\hline & & $\mathrm{S}^{2}$ & 2.26 & 2.23 & 1.27 & 53.10 & 49.16 & 44.99 \\
\hline & \multirow{4}{*}{5} & $\mathrm{x}^{-}$ & 35.00 & 34.56 & 34.40 & 38.60 & 35.44 & 35.04 \\
\hline & & $\mathrm{S}^{2}$ & 2.29 & 2.28 & 2.24 & 47.87 & 32.49 & 37.85 \\
\hline \multirow{10}{*}{$\begin{array}{l}\text { Grain filling rate } \\
\qquad(\mathrm{g} / \text { day })\end{array}$} & & $\mathrm{X}^{-}$ & 0.55 & 0.61 & 0.75 & 0.70 & 0.68 & 0.57 \\
\hline & & $\mathrm{S}^{2}$ & 0.005 & 0.003 & 0.004 & 0.07 & 0.06 & 0.05 \\
\hline & \multirow{2}{*}{2} & $\mathrm{X}^{-}$ & 0.55 & 0.48 & 0.67 & 0.62 & 0.52 & 0.49 \\
\hline & & $\mathrm{S}^{2}$ & 0.006 & 0.002 & 0.003 & 0.08 & 0.06 & 0.06 \\
\hline & \multirow{2}{*}{3} & $\mathrm{X}^{-}$ & 0.56 & 0.30 & 0.60 & 0.54 & 0.49 & 0.44 \\
\hline & & $\mathrm{S}^{2}$ & 0.005 & 0.004 & 0.005 & 0.09 & 0.08 & 0.07 \\
\hline & \multirow{2}{*}{4} & $\mathrm{X}^{-}$ & 0.46 & 0.32 & 0.52 & 0.50 & 0.43 & 0.40 \\
\hline & & $\mathrm{S}^{2}$ & 0.002 & 0.004 & 0.004 & 0.11 & 0.09 & 0.10 \\
\hline & \multirow{2}{*}{5} & $\mathrm{X}^{-}$ & 0.55 & 0.59 & 0.75 & 0.66 & 0.55 & 0.49 \\
\hline & & $\mathrm{S}^{2}$ & 0.002 & 0.003 & 0.003 & 0.08 & 0.07 & 0.07 \\
\hline
\end{tabular}


TABLE 2. Cont. Mean $\left(\mathrm{X}^{-}\right)$and variance $\left(\mathrm{S}^{2}\right)$ of the six populations of the five crosses for plant height, spike length, number of grains/spike and number of spikes/plant

\begin{tabular}{|c|c|c|c|c|c|c|c|c|}
\hline Trait & Cross & $\begin{array}{l}\text { Statistical } \\
\text { Parameter }\end{array}$ & P1 & P2 & F1 & F2 & BC1 & BC2 \\
\hline \multirow{10}{*}{$\begin{array}{l}\text { Plant height } \\
(\mathrm{cm})\end{array}$} & \multirow[b]{2}{*}{1} & $\mathrm{X}^{-}$ & 103.50 & 99.00 & 111.00 & 108.02 & 102.04 & 111.44 \\
\hline & & $\mathrm{S}^{2}$ & 16.12 & 22.55 & 14.48 & 97.95 & 89.71 & 80.41 \\
\hline & \multirow{2}{*}{2} & $\mathrm{x}^{-}$ & 105.60 & 90.50 & 106.50 & 108.04 & 106.32 & 104.28 \\
\hline & & $\mathrm{S}^{2}$ & 17.83 & 23.36 & 16.81 & 120.84 & 101.82 & 112.61 \\
\hline & \multirow{2}{*}{3} & $\mathrm{X}^{-}$ & 107.80 & 64.10 & 79.30 & 82.32 & 88.00 & 75.76 \\
\hline & & $\mathrm{S}^{2}$ & 17.68 & 13.75 & 21.80 & 130.31 & 120.32 & 113.54 \\
\hline & \multirow{3}{*}{4} & $x^{-}$ & 90.10 & 64.90 & 82.00 & 94.86 & 88.00 & 74.20 \\
\hline & & $\mathrm{S}^{2}$ & 20.64 & 14.78 & 18.97 & 182.73 & 164.51 & 162.92 \\
\hline & & $\mathrm{x}^{-}$ & 83.80 & 96.30 & 103.00 & 100.70 & 103.16 & 101.88 \\
\hline & 5 & $\mathrm{~S}^{2}$ & 19.61 & 24.01 & 13.45 & 127.98 & 120.22 & 114.89 \\
\hline \multirow{10}{*}{$\begin{array}{l}\text { Spike length } \\
\quad(\mathrm{cm})\end{array}$} & \multirow{2}{*}{1} & $\mathrm{x}^{-}$ & 6.70 & 7.00 & 7.50 & 7.18 & 6.12 & 6.22 \\
\hline & & $\mathrm{S}^{2}$ & 0.13 & 0.12 & 0.17 & 1.79 & 1.16 & 1.57 \\
\hline & \multirow[b]{2}{*}{2} & $\mathrm{x}^{-}$ & 6.90 & 6.80 & 7.50 & 6.52 & 7.24 & 7.04 \\
\hline & & $\mathrm{S}^{2}$ & 0.11 & 0.18 & 0.16 & 1.87 & 1.73 & 1.42 \\
\hline & \multirow{2}{*}{3} & $\mathrm{x}^{-}$ & 6.50 & 5.60 & 7.20 & 5.70 & 6.12 & 5.72 \\
\hline & & $\mathrm{S}^{2}$ & 0.14 & 0.15 & 0.18 & 1.49 & 1.33 & 1.58 \\
\hline & \multirow{2}{*}{4} & $x^{-}$ & 7.70 & 5.90 & 7.60 & 6.58 & 7.04 & 5.88 \\
\hline & & $\mathrm{S}^{2}$ & 0.12 & 0.13 & 0.16 & 1.97 & 1.42 & 1.46 \\
\hline & \multirow{2}{*}{5} & $\mathrm{X}^{-}$ & 5.40 & 7.30 & 8.10 & 5.86 & 7.32 & 5.72 \\
\hline & & $\mathrm{S}^{2}$ & 0.15 & 0.11 & 0.11 & 1.92 & 1.41 & 1.74 \\
\hline \multirow{8}{*}{$\begin{array}{l}\text { Number of grains / } \\
\text { spike } \\
\text { (grain) }\end{array}$} & \multirow{2}{*}{1} & $\mathrm{X}^{-}$ & 56.80 & 56.40 & 62.40 & 56.01 & 56.28 & 55.92 \\
\hline & & $\mathrm{S}^{2}$ & 3.82 & 3.94 & 3.74 & 42.26 & 31.80 & 34.21 \\
\hline & \multirow{2}{*}{2} & $\mathrm{x}^{-}$ & 57.60 & 55.80 & 62.40 & 51.12 & 56.64 & 54.72 \\
\hline & & $\mathrm{S}^{2}$ & 3.24 & 3.82 & 3.39 & 42.02 & 36.50 & 33.47 \\
\hline & \multirow{3}{*}{3} & $\mathrm{X}^{-}$ & 57.00 & 43.80 & 57.60 & 43.92 & 44.88 & 46.32 \\
\hline & & $\mathrm{S}^{2}$ & 4.31 & 5.22 & 4.33 & 48.31 & 38.30 & 42.82 \\
\hline & & $\mathrm{X}^{-}$ & 58.20 & 43.40 & 59.40 & 53.64 & 53.52 & 49.04 \\
\hline & 4 & $\mathrm{~S}^{2}$ & 3.82 & 5.30 & 5.70 & 67.05 & 52.31 & 59.28 \\
\hline \multirow{12}{*}{$\begin{array}{c}\text { Number of spikes } \\
\text { / plant } \\
\text { (spike) }\end{array}$} & \multirow{2}{*}{5} & $\mathrm{x}^{-}$ & 51.00 & 57.00 & 61.20 & 49.92 & 57.36 & 48.96 \\
\hline & & $\mathrm{S}^{2}$ & 4.31 & 3.76 & 3.96 & 55.54 & 49.91 & 47.07 \\
\hline & \multirow[b]{2}{*}{1} & $\mathrm{X}^{-}$ & 14.10 & 12.20 & 14.90 & 13.34 & 14.00 & 12.16 \\
\hline & & $\mathrm{S}^{2}$ & 1.46 & 1.24 & 1.16 & 27.79 & 25.05 & 22.43 \\
\hline & \multirow[b]{2}{*}{2} & $\mathrm{x}^{-}$ & 14.40 & 16.10 & 16.70 & 15.52 & 15.06 & 13.60 \\
\hline & & $\mathrm{S}^{2}$ & 1.51 & 1.13 & 2.01 & 39.67 & 35.90 & 30.42 \\
\hline & \multirow[b]{2}{*}{3} & $\mathrm{X}^{-}$ & 14.70 & 15.20 & 17.20 & 16.40 & 17.08 & 13.76 \\
\hline & & $\mathrm{S}^{2}$ & 1.49 & 1.42 & 1.17 & 35.48 & 28.13 & 28.91 \\
\hline & \multirow{2}{*}{4} & $\mathrm{x}^{-}$ & 11.60 & 15.10 & 16.50 & 17.56 & 15.28 & 14.84 \\
\hline & & $\mathrm{S}^{2}$ & 1.46 & 1.36 & 1.74 & 33.78 & 28.18 & 27.62 \\
\hline & \multirow{2}{*}{5} & $\mathrm{X}^{-}$ & 15.30 & 12.30 & 19.50 & 16.54 & 14.96 & 16.24 \\
\hline & & $\mathrm{S}^{2}$ & 1.53 & 1.22 & 2.05 & 30.49 & 25.44 & 27.27 \\
\hline
\end{tabular}

Egypt. J. Sus. Agric. Sci . 43, No. 3 (2017) 
TABLE 2. Cont. Mean $\left(\mathrm{X}^{-}\right)$and variance $\left(\mathrm{S}^{2}\right)$ of the six populations of the five crosses for 100 -grain weight and grain yield / plant

\begin{tabular}{|c|c|c|c|c|c|c|c|c|}
\hline Trait & Cross & $\begin{array}{l}\text { Statistical } \\
\text { Parameter }\end{array}$ & P1 & P2 & F1 & F2 & BC1 & BC2 \\
\hline \multirow{8}{*}{$\begin{array}{l}\text { 100-grain weight } \\
(\mathrm{g})\end{array}$} & \multirow{2}{*}{1} & $\mathrm{X}^{-}$ & 4.72 & 5.25 & 5.87 & 4.69 & 4.69 & 4.60 \\
\hline & & $\mathrm{S}^{2}$ & 0.05 & 0.03 & 0.04 & 0.57 & 0.46 & 0.49 \\
\hline & \multirow{2}{*}{2} & $\mathrm{X}^{-}$ & 4.68 & 5.44 & 5.73 & 4.98 & 4.61 & 4.97 \\
\hline & & $\mathrm{S}^{2}$ & 0.04 & 0.02 & 0.03 & 0.62 & 0.50 & 0.55 \\
\hline & \multirow{2}{*}{3} & $X^{-}$ & 4.71 & 3.76 & 4.89 & 4.29 & 4.25 & 4.17 \\
\hline & & $\mathrm{S}^{2}$ & 0.04 & 0.04 & 0.04 & 0.63 & 0.58 & 0.54 \\
\hline & \multirow{2}{*}{4} & $X^{-}$ & 4.93 & 3.82 & 4.76 & 4.57 & 4.90 & 4.47 \\
\hline & & $\mathrm{S}^{2}$ & 0.03 & 0.04 & 0.03 & 0.55 & 0.48 & 0.46 \\
\hline \multirow{12}{*}{$\begin{array}{l}\text { Grain yield / plant } \\
(\mathrm{g})\end{array}$} & \multirow{2}{*}{5} & $X^{-}$ & 4.57 & 5.20 & 5.82 & 4.62 & 4.76 & 4.55 \\
\hline & & $\mathrm{S}^{2}$ & 0.05 & 0.03 & 0.05 & 0.50 & 0.46 & 0.45 \\
\hline & \multirow{2}{*}{1} & $\mathrm{X}^{-}$ & 21.58 & 20.81 & 24.66 & 20.76 & 19.93 & 17.46 \\
\hline & & $\mathrm{S}^{2}$ & 4.29 & 5.59 & 3.09 & 52.25 & 47.94 & 42.48 \\
\hline & \multirow{2}{*}{2} & $X^{-}$ & 20.60 & 15.16 & 21.95 & 18.48 & 18.35 & 15.18 \\
\hline & & $\mathrm{S}^{2}$ & 4.33 & 4.87 & 3.95 & 59.04 & 49.05 & 50.05 \\
\hline & \multirow{2}{*}{3} & $X^{-}$ & 21.65 & 11.40 & 22.41 & 17.85 & 17.76 & 13.51 \\
\hline & & $\mathrm{S}^{2}$ & 4.35 & 3.46 & 3.40 & 60.73 & 52.04 & 54.25 \\
\hline & \multirow{2}{*}{4} & $X^{-}$ & 15.48 & 11.95 & 19.05 & 18.82 & 17.75 & 16.53 \\
\hline & & $\mathrm{S}^{2}$ & 4.68 & 3.42 & 3.60 & 54.78 & 48.00 & 44.35 \\
\hline & \multirow{2}{*}{5} & $\mathrm{X}^{-}$ & 19.25 & 20.52 & 25.73 & 21.08 & 19.40 & 17.31 \\
\hline & & $\mathrm{S}^{2}$ & 3.97 & 5.53 & 3.06 & 69.69 & 63.97 & 62.86 \\
\hline
\end{tabular}

\section{Gene effects}

These data were used to calculate the scaling test and six parameters according to Gamble procedure (1962). At least, one of the scales (A, $\mathrm{B}$ and $\mathrm{C}$ ) was significant in the six crosses for all studied traits. However, the significance of any one of the scales reveals the presence of nonallelic interaction as pointed out in Table 3.

Estimates of gene effects calculated from the six-parameter model of the generation mean analysis are presented in Table 3. Desirable values of additive gene effects were obtained from crosses no. 3 and 4 for plant height, 3, 4 and 5 for spike length, 2, 4 and 5 for number of grains/spike, 3 for number of spikes/plant, 4 for 100-grain weight and 1, 2 and 3 for grain yield/plant. This assures the enhancing effect of additive variance in the inheritance of these traits. These results indicated the potentiality of improving the performance of these traits using pedigree selection as reported by Mansour (2012) and Mansour et al. (2014).

However, dominance plays greater role in inheritance of grain filling period, spike length and no. of grains/spike, additive $\mathrm{x}$ additive plays greater role in inheritance of grain filling period, additive $\mathrm{x}$ dominance plays greater role in inheritance of days to heading and maturiety and dominance $x$ dominance plays greater role in inheritance of most yeild traits. It could be observed that when additive effects are larger than non-additive ones, it is suggested that selection would be effective in early segregating generations, while if the non-additive portion are larger than additive ones, the improvement of the characters need intensive selection in the later generations. However, when epistatic effect was significant for a trait, the possibility of obtaining desirable segregates through inter-mating in early segregating generations by breaking undesirable linkage could be available or it is suggested to adopt recurrent selection for handling the above crosses for rapid improvement. These conclusions are in the same line with those reported by Zeng et al. (2001), Eid (2006), Munir et al. (2007), Khattab et al. (2010), Aykuttonk et al. (2011), Mansour (2012), Amin (2013), Ljaz et al. (2013), Mansour et al. (2015), Abaas et al (2016) and Mansour (2016).

Heterosis, potence ratio and inbreeding depression

Heterosis over mid-parent and better parent, potence ratio and inbreeding depression, are presented in Table 4. Significant and highly significant desirable percentages of heterosis relative to mid and better parent with insignificant values for inbreeding depression were exhibited for grain filling period in crosses no. 1, 3 and 5 , plant height in crosses no. 1 and 5, no. of grains/spike in crosses no. 1 and 4, no. of spikes/plant in crosses no. 2, 3 and 4 and grain yield/plant in cross no. 4 .

Egypt. J. Sus. Agric. Sci . 43, No. 3 (2017) 
TABLE 3. Scaling test and type of gene action estimated by generation means of the five crosses for days to heading, days to maturity, grain filling period, grain filling rate and plant height

\begin{tabular}{|c|c|c|c|c|c|c|c|c|c|c|}
\hline \multirow{2}{*}{ Trait } & \multirow{2}{*}{ Cross } & \multicolumn{3}{|c|}{ Scaling test } & \multicolumn{6}{|c|}{ Type of gene action } \\
\hline & & $\mathbf{A}$ & B & C & (m) & (a) & (d) & (aa) & (ad) & (dd) \\
\hline \multirow{4}{*}{$\begin{array}{l}\text { Days to } \\
\text { heading }\end{array}$} & 1 & $-2.52 *$ & $-3.42 * *$ & $-9.63 * *$ & $75.32 * *$ & $6.40^{* *}$ & $8.84 * *$ & 3.69 & 0.45 & 2.25 \\
\hline & 2 & $-2.59 * *$ & $2.14^{*}$ & $-6.21 * *$ & $77.70 * *$ & $2.04 * *$ & $9.05 * *$ & $5.76^{* *}$ & $-2.37 * *$ & $-5.31^{*}$ \\
\hline & 4 & $-4.08 * *$ & $7.42 * *$ & $-6.17 * *$ & $76.58^{* *}$ & $6.40 * *$ & $11.86^{* *}$ & $9.51 * *$ & $-5.75^{* *}$ & $-12.85^{* *}$ \\
\hline & 5 & $-7.82 * *$ & 1.38 & $-5.11 * *$ & $77.93 * *$ & $4.48 * *$ & 1.05 & -1.33 & $-4.60 * *$ & $7.77 * *$ \\
\hline \multirow{3}{*}{$\begin{array}{l}\text { Days to } \\
\text { maturity }\end{array}$} & 1 & $-15.69 * *$ & $-9.05 * *$ & $-20.73 * *$ & $107.38 * *$ & $5.08 * *$ & -2.68 & $-4.01 *$ & $-3.32 * *$ & $28.76^{* *}$ \\
\hline & 4 & $-6.88 * *$ & -0.67 & $-3.88^{*}$ & $113.42 * *$ & $6.92 * *$ & -0.04 & -3.67 & $-3.11 * *$ & $11.22 * *$ \\
\hline & 5 & $-6.34 * *$ & $2.50^{*}$ & $10.93 * *$ & $116.53 * *$ & $4.88 * *$ & $-12.77 * *$ & $-14.77 * *$ & $-4.42 * *$ & $18.61 * *$ \\
\hline & 1 & $-13.17 * *$ & $-5.63 * *$ & $-11.10 * *$ & $32.07 * *$ & -1.32 & $-11.52 * *$ & $-7.71 * *$ & $-3.77 * *$ & $26.51 * *$ \\
\hline Grain & 2 & $-3.78^{*}$ & -1.25 & 0.98 & $33.88^{*} *$ & 1.88 & $-7.82 * *$ & $-6.01 *$ & -1.27 & $11.05 * *$ \\
\hline filling & 3 & $-4.17 *$ & $-13.98 * *$ & 1.36 & $38.22 * *$ & $5.16^{* *}$ & $-20.06 * *$ & $-19.51 * *$ & $4.91 * *$ & $37.66^{* *}$ \\
\hline \multirow{2}{*}{ period } & 4 & -2.80 & $-8.09 * *$ & 2.28 & $36.83 * *$ & 0.52 & $-11.90 * *$ & $-13.17 * *$ & $2.65^{*}$ & $24.06^{* *}$ \\
\hline & 5 & 1.48 & 1.12 & $16.04 * *$ & $38.60 * *$ & 0.40 & $-13.82 * *$ & $-13.44 * *$ & 0.18 & $10.84 *$ \\
\hline $\begin{array}{l}\text { Grain } \\
\text { filling rate }\end{array}$ & 5 & $-0.20 * *$ & $-0.35^{* *}$ & 0.01 & $0.66^{* *}$ & 0.05 & $-0.38 * *$ & $-0.56^{* *}$ & 0.08 & $1.11 * *$ \\
\hline \multirow{5}{*}{$\begin{array}{l}\text { Plant } \\
\text { height }\end{array}$} & 1 & $-10.42 * *$ & $12.88 * *$ & $7.58 * *$ & $108.02 * *$ & $-9.40 * *$ & 4.63 & -5.12 & $-11.65^{* *}$ & 2.66 \\
\hline & 2 & 0.54 & $11.56^{* *}$ & $23.06^{* *}$ & $108.04 * *$ & 2.04 & -2.51 & $-10.96 * *$ & $-5.51 * *$ & -1.14 \\
\hline & 3 & $-11.10 * *$ & $8.12^{* *}$ & -1.22 & $82.32 * *$ & $12.24 * *$ & -8.41 & -1.76 & $-9.61 * *$ & 4.74 \\
\hline & 4 & 3.90 & 1.50 & $60.44 * *$ & $94.86^{* *}$ & $13.80 * *$ & $-50.54 * *$ & $-55.04 * *$ & 1.20 & $49.64 * *$ \\
\hline & 5 & $19.52 * *$ & 4.46 & $16.70 * *$ & $100.70 * *$ & 1.28 & $20.23 * *$ & 7.28 & $7.53^{* *}$ & $-31.26 * *$ \\
\hline
\end{tabular}

$(*)$ and $(* *)$ significant at 0.05 and 0.01 levels of probability, respectively

TABLE 3. Cont. Scaling test and type of gene action estimated by generation means of the five crosses for spike length, number of grains / spike, number of spikes / plant, 100-grain weight and grain yield / plant

\begin{tabular}{|c|c|c|c|c|c|c|c|c|c|c|}
\hline \multirow{2}{*}{ Trait } & \multirow{2}{*}{ Cross } & \multicolumn{3}{|c|}{ Scaling test } & \multicolumn{6}{|c|}{ Type of gene action } \\
\hline & & $\mathbf{A}$ & B & C & (m) & (a) & (d) & (aa) & (ad) & (dd) \\
\hline \multirow{5}{*}{$\begin{array}{l}\text { Spike } \\
\text { length }\end{array}$} & 1 & $-1.96 * *$ & $-2.06 * *$ & 0.02 & $7.18 * *$ & -0.10 & $-3.39 * *$ & $-4.04 * *$ & 0.05 & $8.06 * *$ \\
\hline & 2 & 0.08 & -0.22 & $-2.62 * *$ & $6.52 * *$ & 0.20 & $3.13 * *$ & $2.48 * *$ & 0.15 & $-2.34 * *$ \\
\hline & 3 & $-1.46^{* *}$ & $-1.36 * *$ & $-3.70 * *$ & $5.70 * *$ & $0.40 *$ & $2.03 * *$ & 0.88 & -0.05 & $1.94 *$ \\
\hline & 4 & $-1.22 * *$ & $-1.74 * *$ & $-2.48 * *$ & $6.58 * *$ & $1.16^{* *}$ & 0.32 & -0.48 & 0.26 & $3.44 * *$ \\
\hline & 5 & $1.14 * *$ & $-3.96 * *$ & $-5.46 * *$ & $5.86 * *$ & $1.60 * *$ & $4.39 * *$ & $2.64 * *$ & $2.55 * *$ & 0.18 \\
\hline \multirow{5}{*}{$\begin{array}{l}\text { Number } \\
\text { of grains / } \\
\text { spike }\end{array}$} & 1 & $-8.64 * *$ & $-8.96 * *$ & $-13.96 * *$ & $56.01 * *$ & 0.36 & 2.16 & -3.64 & 0.16 & $21.24 * *$ \\
\hline & 2 & $-6.72 * *$ & $-8.76 * *$ & $-33.72 * *$ & $51.12 * *$ & $1.92 *$ & $23.94 * *$ & $18.24 * *$ & 1.02 & -2.76 \\
\hline & 3 & $-24.84 * *$ & $-8.76 * *$ & $-40.32 * *$ & $43.92 * *$ & -1.44 & $13.92 * *$ & $6.72 *$ & $-8.04 * *$ & $26.88 * *$ \\
\hline & 4 & $-10.56 * *$ & $-4.72 *$ & $-5.84 * *$ & $53.64 * *$ & $4.48 * *$ & -0.84 & $-9.44 * *$ & $-2.92 *$ & $24.72 * *$ \\
\hline & 5 & 2.52 & $-20.28 * *$ & $-30.72 * *$ & $49.92 * *$ & $8.40 * *$ & $20.16^{* *}$ & $12.96^{* *}$ & $11.40 * *$ & 4.80 \\
\hline \multirow{5}{*}{$\begin{array}{c}\text { Number } \\
\text { of spikes / } \\
\text { plant }\end{array}$} & 1 & -1.00 & $-2.78^{*}$ & $-2.74 *$ & $13.34 * *$ & $1.84 *$ & 0.71 & -1.04 & 0.89 & 4.82 \\
\hline & 2 & $-3.90 * *$ & $-2.68^{*}$ & -1.82 & $15.52 * *$ & -1.46 & -3.31 & $-4.76^{*}$ & -0.61 & $11.34 * *$ \\
\hline & 3 & 2.26 & $-4.88 * *$ & 1.30 & $16.40 * *$ & $3.32 * *$ & -1.67 & -3.92 & $3.57 * *$ & 6.54 \\
\hline & 4 & 2.46 & -1.92 & $10.54 * *$ & $17.56 * *$ & 0.44 & $-6.85 * *$ & $-10.00 * *$ & $2.19 *$ & $9.46^{*}$ \\
\hline & 5 & $-4.88 * *$ & 0.68 & -0.44 & $16.54 * *$ & -1.28 & 1.94 & -3.76 & $-2.78 * *$ & $7.96^{*}$ \\
\hline \multirow{5}{*}{$\begin{array}{l}\text { 100-grain } \\
\text { weight }\end{array}$} & 1 & $-1.22 * *$ & $-1.91 * *$ & $-2.94 * *$ & $4.69 * *$ & 0.08 & $0.70 *$ & -0.19 & $0.35^{* *}$ & $3.32 * *$ \\
\hline & 2 & $-1.19 * *$ & $-1.23 * *$ & $-1.66^{* *}$ & $4.98 * *$ & $-0.36 * *$ & -0.09 & $-0.76^{*}$ & 0.02 & $3.18 * *$ \\
\hline & 3 & $-1.11 * *$ & -0.32 & $-1.10 * *$ & $4.29 * *$ & 0.08 & 0.33 & -0.32 & $-0.40 * *$ & $1.75 * *$ \\
\hline & 4 & 0.12 & $0.37 *$ & 0.04 & $4.57 * *$ & $0.43 * *$ & $0.83 * *$ & 0.45 & -0.13 & -0.94 \\
\hline & 5 & $-0.88 * *$ & $-1.92 * *$ & $-2.92 * *$ & $4.62 * *$ & 0.21 & $1.06 * *$ & 0.12 & $0.52 * *$ & $2.68 * *$ \\
\hline \multirow{5}{*}{$\begin{array}{l}\text { Grain yield } \\
\text { / plant }\end{array}$} & 1 & $-6.38 * *$ & $-10.55^{* *}$ & $-8.67 * *$ & $20.76 * *$ & $2.47 *$ & -4.80 & $-8.26 * *$ & 2.09 & $25.19 * *$ \\
\hline & 2 & $-5.85 * *$ & $-6.75 * *$ & $-5.74 * *$ & $18.48 * *$ & $3.17 * *$ & -2.79 & $-6.86^{*}$ & 0.45 & $19.46^{* *}$ \\
\hline & 3 & $-8.53 * *$ & $-6.79 * *$ & $-6.47 * *$ & $17.85 * *$ & $4.25^{* *}$ & -2.97 & $-8.86 * *$ & -0.87 & $24.18 * *$ \\
\hline & 4 & 0.98 & 2.05 & $9.75 * *$ & $18.82 * *$ & 1.23 & -1.39 & $-6.72 *$ & -0.54 & 3.70 \\
\hline & 5 & $-6.17 * *$ & $-11.63^{* *}$ & $-6.90 * *$ & $21.08 * *$ & 2.09 & -5.06 & $-10.90 * *$ & $2.73 *$ & $28.70 * *$ \\
\hline
\end{tabular}

$(*)$ and $(* *)$ significant at 0.05 and 0.01 levels of probability, respectively

Egypt. J. Sus. Agric. Sci. 43, No. 3 (2017) 
TABLE 4. Heterosis, inbreeding depression and potence ratio in five crosses for all studied traits

\begin{tabular}{|c|c|c|c|c|c|c|c|c|c|c|c|}
\hline \multirow{2}{*}{ Trait } & \multirow{2}{*}{ Cross } & \multicolumn{2}{|c|}{ Heterosis } & \multirow{2}{*}{ ID } & \multirow{2}{*}{ PR\% } & \multirow{2}{*}{ Traits } & \multirow{2}{*}{ Cross } & \multicolumn{2}{|c|}{ Heterosis } & \multirow{2}{*}{ ID } & \multirow{2}{*}{ PR\% } \\
\hline & & MP & BP & & & & & MP & BP & & \\
\hline \multirow{5}{*}{$\begin{array}{l}\text { Days to } \\
\text { heading }\end{array}$} & 1 & $6.85 * *$ & $16.04 * *$ & 6.21 & 0.87 & \multirow{5}{*}{$\begin{array}{l}\text { Spike } \\
\text { length }\end{array}$} & 1 & $9.49 * *$ & $7.14 * *$ & $4.27 * *$ & 4.33 \\
\hline & 2 & $4.25 * *$ & $10.52^{* *}$ & 3.96 & 0.75 & & 2 & $9.49 * *$ & $8.70^{* *}$ & $13.07 * *$ & 13.00 \\
\hline & 3 & $4.11^{* *}$ & $17.34^{* *}$ & 0.50 & 0.36 & & 3 & $19.01 * *$ & $10.77 * *$ & $20.83^{* *}$ & 2.56 \\
\hline & 4 & $3.05 * *$ & $22.38 * *$ & 3.43 & 0.19 & & 4 & $11.76^{* *}$ & $-1.30 * *$ & $13.42 * *$ & 0.89 \\
\hline & 5 & $3.05 * *$ & $16.62 * *$ & 3.07 & 0.26 & & 5 & $27.56^{* *}$ & $10.96^{* *}$ & $27.65^{* *}$ & 1.84 \\
\hline \multirow{5}{*}{$\begin{array}{l}\text { Days to } \\
\text { maturity }\end{array}$} & 1 & $1.19 * *$ & $9.40 * *$ & 5.17 & 0.16 & \multirow{5}{*}{$\begin{array}{c}\text { Number } \\
\text { of grains / } \\
\text { spike }\end{array}$} & 1 & $10.25 * *$ & $9.86^{* *}$ & 10.24 & 29.00 \\
\hline & 2 & $1.32 * *$ & $8.64 * *$ & 1.80 & 0.20 & & 2 & $10.05^{* *}$ & $8.33^{* *}$ & $18.08 * *$ & 6.33 \\
\hline & 3 & $2.21 * *$ & $10.67 * *$ & -0.20 & 0.29 & & 3 & $14.29 * *$ & $1.05^{* *}$ & $23.75^{* *} *$ & 1.09 \\
\hline & 4 & $3.22 * *$ & $13.31 * *$ & 2.40 & 0.36 & & 4 & $16.93 * *$ & $2.06^{* *}$ & 9.70 & 1.16 \\
\hline & 5 & $1.77 * *$ & $10.92^{* *}$ & -1.51 & 0.22 & & 5 & $13.33 * *$ & $7.37 * *$ & $18.43 *$ & 2.40 \\
\hline \multirow{5}{*}{$\begin{array}{l}\text { Grain } \\
\text { filling } \\
\text { period }\end{array}$} & 1 & $-10.4 * *$ & $-3.98 * *$ & 2.63 & -1.56 & \multirow{5}{*}{$\begin{array}{c}\text { Number } \\
\text { of spikes } \\
\text { / plant }\end{array}$} & 1 & $13.31 * *$ & $5.67 * *$ & $10.47 *$ & 1.84 \\
\hline & 2 & $-5.24 * *$ & $4.25 * *$ & -3.51 & -0.58 & & 2 & $9.51 * *$ & $3.73 * *$ & 7.07 & 1.71 \\
\hline & 3 & $-1.45^{* *}$ & $-0.79 *$ & -1.64 & -2.18 & & 3 & $15.05 * *$ & $13.16^{* *}$ & 4.65 & 9.00 \\
\hline & 4 & $3.58 * *$ & $10.15^{* *}$ & 0.18 & 0.60 & & 4 & $23.60 * *$ & $9.27 * *$ & -6.42 & 1.80 \\
\hline & 5 & $-1.09 * *$ & $-0.46^{* *}$ & -12.21 & -1.73 & & 5 & $41.30 * *$ & $27.45^{* *}$ & $15.18 * *$ & 3.80 \\
\hline \multirow{5}{*}{$\begin{array}{l}\text { Grain } \\
\text { filling } \\
\text { rate }\end{array}$} & 1 & $29.41 * *$ & $23.42^{* *}$ & $6.52 * *$ & 6.06 & & 1 & $17.80^{* *}$ & $11.85 * *$ & $20.07 * *$ & 3.35 \\
\hline & 2 & $30.28 * *$ & $22.69 * *$ & $7.54 * *$ & 4.90 & \multirow{4}{*}{$\begin{array}{c}\text { 100-grain } \\
\text { weight }\end{array}$} & 2 & $13.22 * *$ & $5.29 * *$ & $13.09 * *$ & 1.76 \\
\hline & 3 & $37.91 * *$ & $5.76^{* *}$ & $9.40 * *$ & 1.25 & & 3 & $15.44 * *$ & $3.82 * *$ & $12.32 * *$ & 1.38 \\
\hline & 4 & $32.60 * *$ & $11.72 * *$ & $3.15^{* *}$ & 1.74 & & 4 & $8.78 * *$ & $-3.49 * *$ & $3.82 * *$ & 0.69 \\
\hline & 5 & $30.77 * *$ & $25.95 * *$ & $11.74 * *$ & 8.03 & & 5 & $19.12 * *$ & $11.92 * *$ & $20.57 * *$ & 2.97 \\
\hline \multirow{5}{*}{$\begin{array}{l}\text { Plant } \\
\text { height }\end{array}$} & 1 & $9.63 * *$ & $7.25 * *$ & 2.68 & 4.33 & \multirow{5}{*}{$\begin{array}{l}\text { Grain } \\
\text { yield / } \\
\text { plant }\end{array}$} & 1 & $16.35^{* *}$ & $14.27 * *$ & $15.82 *$ & 9.00 \\
\hline & 2 & $8.62 * *$ & 0.85 & -1.45 & 1.12 & & 2 & $22.75^{* *} *$ & $6.55^{* *}$ & $15.81 *$ & 1.50 \\
\hline & 3 & $-7.74 * *$ & $-26.4 * *$ & -3.81 & -0.30 & & 3 & $35.63^{* *}$ & $3.53 * *$ & $20.35^{*}$ & 1.15 \\
\hline & 4 & $5.81 * *$ & $-8.99 * *$ & -15.68 & 0.36 & & 4 & $38.90 * *$ & $23.06^{* *}$ & 1.21 & 3.02 \\
\hline & 5 & $14.38 * *$ & $6.96^{* *}$ & 2.23 & 2.07 & & 5 & $29.38 * *$ & $25.37 * *$ & $18.06^{*}$ & 9.18 \\
\hline
\end{tabular}

$(*)$ and $(* *)$ significant at 0.05 and 0.01 levels of probability, respectively

For potence ratio, results showed over dominance for all studied traits in all crosses except for days to heading and matureity in all crosses, grain filling period in crosses no. 2 and 4, plant height in crosses no 3 and 4, spike length and 100-grain weight in cross no. 4. Similar findings were also reported by Mahmoud, Badeaa (2006), Eid (2006), Munir et al. (2007), ElAkhdar (2011), Amin (2013), El-Refaey and Abd El-Razek (2013) Mansour et al. (2015), Abaas et al (2016) and Mansour (2016).

Heritability in broad and narrow-senses and genetic advance

Heritability in broad and narrow-senses and genetic advance, are presented in Table 5. Broad sense heritability values $\left(\mathrm{H}^{2}\right)$ estimates were generally higher than the corresponding narrowsense heritability $\left(\mathrm{h}^{2}\right)$, indicating the presence of non-additive gene action. Broad-sense heritability values were high in all crosses for all studied traits and ranged from 82.74 to $98.00 \%$ in cross no. 1 for plant height and days to heading, respectively.
Narrow-sense heritability ranged from low to medium in most cases due to the opposite directions of dominance and dominance $\mathrm{x}$ dominance effects with values; 5.17 to $53.96 \%$ for spike length in crosses no. 3 and 4 , respectively. Genetic advance from selection ranged from $1.92 \%$ in cross no. 2 for days to maturity to $49.27 \%$ in cross no. 2 for grain filling rate. High percentage of expected genetic advance would help breeder in improving the trait of interest via few cycles of selection. These results are in good agreement with those reported by Eid (2006), Munir et al. (2007), Khattab et al. (2010), ElAkhdar (2011), Aykuttonk et al. (2011), Mansour (2012), Amin (2013), El-Refaey \& Abd El-Razek (2013), Mansour et al. (2015), Abaas et al (2016) and Mansour (2016).

Generally, the most promising crosses were the two crosses 1 and 5 for earliness and crosses no. 2, 3 and 4 for grain yield and were found to be higher in magnitude, which expressed high genetic advance associated with high heritability and would be of interest in breeding programs for improving the studied traits in barley.

Egypt. J. Sus. Agric. Sci . 43, No. 3 (2017) 
TABLE 5. Heritability percentage in broad $\left(\mathrm{H}^{2}\right)$ and narrow $\left(\mathrm{h}^{2}\right)$ senses and expected genetic advance from selection $(\Delta \mathrm{g})$ of the five crosses for all studied traits

\begin{tabular}{|c|c|c|c|c|c|c|c|c|c|c|c|}
\hline & \multirow[t]{2}{*}{ Cross } & \multicolumn{2}{|c|}{ Heritability } & \multicolumn{2}{|c|}{$\begin{array}{c}\text { Genetic } \\
\text { advance }\end{array}$} & \multirow[t]{2}{*}{ Traits } & \multirow[t]{2}{*}{ Cross } & \multicolumn{2}{|c|}{ Heritability } & \multicolumn{2}{|c|}{ Genetic advance } \\
\hline & & $\mathbf{H}^{2}$ & $h^{2}$ & $\Delta \mathrm{g}$ & $\Delta \mathrm{g} \%$ & & & $\mathbf{H}^{2}$ & $h^{2}$ & $\Delta \mathrm{g}$ & $\Delta \mathrm{g} \%$ \\
\hline \multirow{5}{*}{$\begin{array}{l}\text { Days to } \\
\text { heading }\end{array}$} & 1 & 98.00 & 33.92 & 3.59 & 4.77 & \multirow{5}{*}{$\begin{array}{l}\text { Spike } \\
\text { length }\end{array}$} & 1 & 91.76 & 47.62 & 1.31 & 18.28 \\
\hline & 2 & 96.38 & 39.25 & 3.37 & 4.34 & & 2 & 91.84 & 31.94 & 0.90 & 13.80 \\
\hline & 3 & 96.53 & 30.76 & 3.46 & 4.55 & & 3 & 89.13 & 5.17 & 0.13 & 2.28 \\
\hline & 4 & 96.52 & 38.36 & 3.36 & 4.39 & & 4 & 92.77 & 53.96 & 1.56 & 23.71 \\
\hline & 5 & 94.24 & 44.68 & 3.67 & 4.71 & & 5 & 93.75 & 35.68 & 1.02 & 17.38 \\
\hline \multirow{6}{*}{$\begin{array}{l}\text { Days to } \\
\text { maturity }\end{array}$} & 1 & 93.23 & 26.68 & 2.86 & 2.67 & & 1 & 90.98 & 43.80 & 5.87 & 10.47 \\
\hline & 2 & 91.99 & 22.85 & 2.14 & 1.92 & \multirow{5}{*}{$\begin{array}{l}\text { Number } \\
\text { of grains / } \\
\text { spike }\end{array}$} & 2 & 91.77 & 33.48 & 4.47 & 8.75 \\
\hline & 3 & 94.17 & 29.06 & 3.25 & 2.84 & & 3 & 90.59 & 32.11 & 4.60 & 10.47 \\
\hline & 4 & 94.71 & 33.95 & 4.42 & 3.89 & & 4 & 92.35 & 33.59 & 5.67 & 10.56 \\
\hline & 5 & 93.58 & 39.96 & 4.37 & 3.75 & & 5 & 92.80 & 25.38 & 3.90 & 7.81 \\
\hline & 1 & 92.72 & 34.53 & 4.18 & 13.04 & & 1 & 95.48 & 29.12 & 3.16 & 23.71 \\
\hline \multirow{4}{*}{$\begin{array}{l}\text { Grain } \\
\text { filling } \\
\text { period }\end{array}$} & 2 & 93.89 & 39.06 & 5.41 & 15.98 & \multirow{4}{*}{$\begin{array}{l}\text { Number } \\
\text { of spikes } \\
\text { / plant }\end{array}$} & 2 & 95.80 & 32.82 & 4.26 & 27.44 \\
\hline & 3 & 95.88 & 21.88 & 3.51 & 9.18 & & 3 & 96.30 & 39.24 & 4.81 & 29.36 \\
\hline & 4 & 96.69 & 22.68 & 3.40 & 9.24 & & 4 & 95.34 & 34.81 & 4.17 & 23.74 \\
\hline & 5 & 95.27 & 53.07 & 7.56 & 19.60 & & 5 & 94.38 & 27.14 & 3.09 & 18.66 \\
\hline \multirow{5}{*}{$\begin{array}{l}\text { Grain } \\
\text { filling } \\
\text { rate }\end{array}$} & 1 & 94.29 & 49.17 & 0.27 & 38.28 & & 1 & 92.98 & 33.33 & 0.52 & 11.05 \\
\hline & 2 & 95.55 & 52.85 & 0.31 & 49.27 & \multirow{4}{*}{$\begin{array}{l}\text { 100-grain } \\
\text { weight }\end{array}$} & 2 & 94.89 & 31.37 & 0.51 & 10.22 \\
\hline & 3 & 94.72 & 33.33 & 0.21 & 38.15 & & 3 & 93.65 & 22.22 & 0.36 & 8.47 \\
\hline & 4 & 96.82 & 27.27 & 0.19 & 37.27 & & 4 & 94.09 & 29.09 & 0.44 & 9.72 \\
\hline & 5 & 96.56 & 22.35 & 0.13 & 19.73 & & 5 & 91.00 & 18.00 & 0.26 & 5.67 \\
\hline \multirow{5}{*}{$\begin{array}{l}\text { Plant } \\
\text { height }\end{array}$} & 1 & 82.74 & 26.32 & 5.37 & 4.97 & \multirow{5}{*}{$\begin{array}{l}\text { Grain } \\
\text { yield / } \\
\text { plant }\end{array}$} & 1 & 92.32 & 26.95 & 4.01 & 19.33 \\
\hline & 2 & 84.52 & 22.55 & 5.11 & 4.73 & & 2 & 92.76 & 32.15 & 5.09 & 27.54 \\
\hline & 3 & 85.61 & 20.54 & 4.83 & 5.87 & & 3 & 93.98 & 24.98 & 4.01 & 22.47 \\
\hline & 4 & 89.96 & 20.81 & 5.79 & 6.11 & & 4 & 93.02 & 31.42 & 4.79 & 25.45 \\
\hline & 5 & 86.22 & 16.29 & 3.80 & 3.77 & & 5 & 94.40 & 18.01 & 3.10 & 14.69 \\
\hline
\end{tabular}

\section{References}

Abaas, Sh. I., El-Shawy, E.E. and Mansour, M. (2016) Genetic parameters for yield and its components in barley. Egypt. J. Plant Breed., 20 (1), 135-149.

Abdel-Nour, Nadya A.R. (2011) Genetic studies on grain yield and earliness components in bread wheat of different photo thermal response. Egypt. J. Agric. Res., 89 (4).

Allard, A.M. (1960) Principles of Plant Breeding. John Wiley and Sons. Inc. NY, U. S. A., p. 92.

Amin, I. A. (2013) Genetic behavior of some agronomic traits in two durum wheat crosses under heat stress. Alex. J. Agric. Res., 58 (1), 53-66.

Aykuttonk, F., Ilker, E. and Tosun, M. (2011) Quantitative inheritance of some wheat agronomic traits. Bulgarian J. Agric. Sci. 17 (6), 783-788.

Egypt. J. Sus. Agric. Sci . 43, No. 3 (2017)
Eid, A.A. (2006) Breeding studies on some barley diseases. Ph.D. Thesis, Fac. Agric. Menofiya. Univ., Egypt.

El-Akhdar, A.A.A. (2011) Genetic studies on yield and its components in some barley crosses. M.Sc. Thesis. Fac., Agric., Kafrelsheikh, Uni., Egypt.

El-Refaey, R.A. and Abd El-Razek, U.A. (2013) Generation mean analysis for yield, its components and quality characteristics in four crosses of Egyption cotton (Gossypuim barbadense L.). Asian J. Crop Sci., 15,153-166.

Gamble, E.E. (1962) Gene effects in corn (Zea mays L.). I-Separtion and relative importance of gene effects for yield. Can. J. Plant Sci., 42, 339-348.

Gebeyehou, G.D.R. Knott, and Baker, R. J. (1982) Rate and duration of grain filling in durum wheat cultivars. Crop. Sci. 22, 337-340. 
Johnson, H.W., Robinson, H. F. and Comstock, R. E. (1955) Estimation of genetic and environmental variability in soybeans. Agron. J., 47, 314-318.

Khattab S. A. M., Esmail, R. M. and Al-Ansary, A. M. F. (2010) Genetical analysis of some quantitative traits in bread weight (Triticum aestivum L.). New York Science Journal, 3 (11), 152-157.

Ljaz,U., Smiullah and Kashif, M. (2013) Genetic study of quantitative trait in spring wheat throught generation mean analysis. American-Eurasian $J$. Agric \& Environ.Sci., 13 (2), 191-197.

Mahmoud, Badeaa, A. M. (2006) Genetic evaluation of some barley traits in crosses under saline and nonsaline conditions. M. Sc. Thesis Fac., Agric., Kafr El-Sheikh, Tanta Univ., Egypt.

Mansour, M.A. (2012) Genetical studies on some barley genotypes and its tolerance to drought. Ph.D. Thesis. Fac., Agric., Tanta, Uni., Egypt.

Mansour, M., Abdel-Hafez, A.G., Amer, Kh.A. and El-Shawy, E.E. (2015) Genetical studies on barley productivity in relation to leaf rust infection. Egypt. J. Agric. Res., 93, 2 (A), 171-185.

Mansour, M. (2016) Genetical analysis of some quantitative traits in barley under saline soil conditions. Proceeding, the Sixth Field Crops Conference, FCRI, ARC, Giza, Egypt 22-23 Nov. 2016, 99-107.
Mansour, M., El-Shawy, E.E. and Abaas, Sh.I. (2016) Genetic improvement of water stress tolerance in some barley genotypes. Egypt. J. Plant Breed., 20 (1),119-134.

Mather, K. (1949) Biometrical Genetics. Dover Publications, Inc., London.

Mather, K., Jinks, J.L. (1982) In: Biometrical Genetics, third ed. Chapman and Hall Ltd., ISBN-10, 0412228904

Miller, P. A., Williams., J. C., Robinson, H. F. and Comstock, R. E. (1958) Estimates of genotypic and environmental variances and covariance in Upland cotton and their implications in selection. Agron. J., 50, 126-131.

Mou, B., Kronstad, W. E. and Saulescu, N. N. (1994) Grain filling parameters and protein content in selected winter wheat population: II. Association. Crop Sci. 34, 838-841.

Munir, M., Chowdhry, M.A. and Ahsan, M. (2007) Generation mean studies in bread wheat under drought condition. Int. J. Agric \& Biology, 9 (2), 282-286.

Zeng, Y., Chen Liang Zeng and Chen, L.Z. (2001) Combining ability and heterosis in forage barley. Indian J. Genet. and Plant Breed., 61 (1), 71-73.

(Received: 28/ 8/ 2017; accepted: $31 / 10 / 2017)$

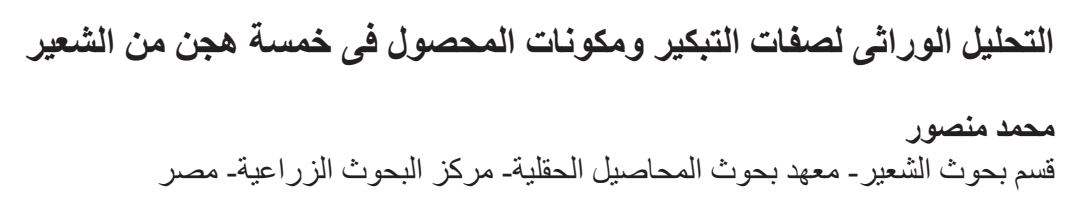

أجريث هذه الدراسة بمزرعة محطة البحوث الزراعية بسخا خلال الثناثة مواسم 14/2013، 15/2014

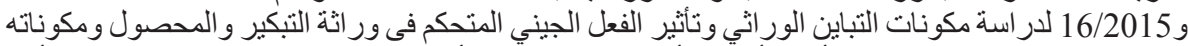

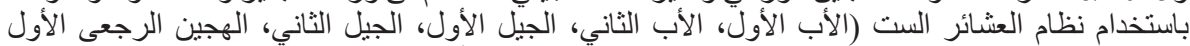

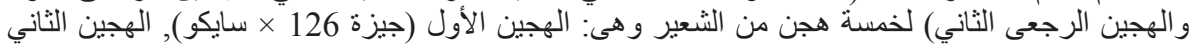

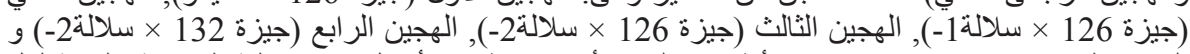

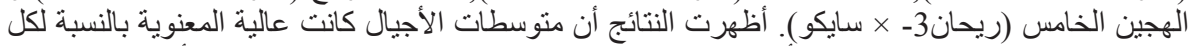

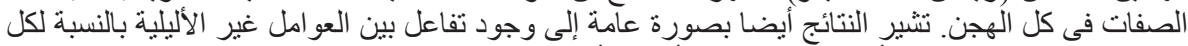

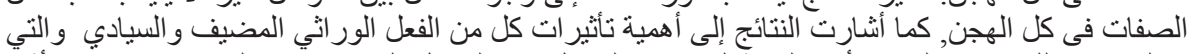

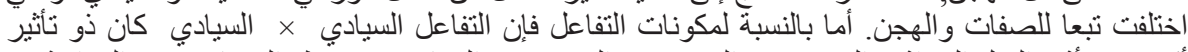

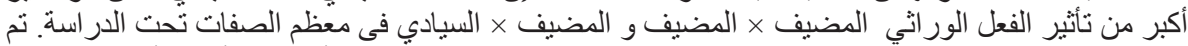

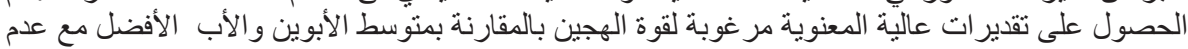

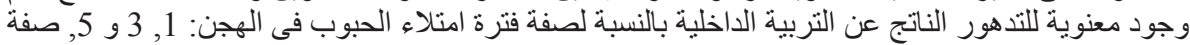

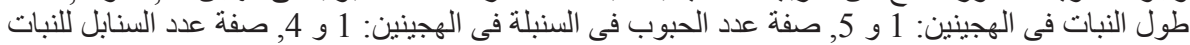

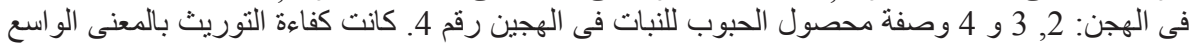

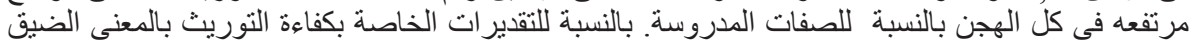

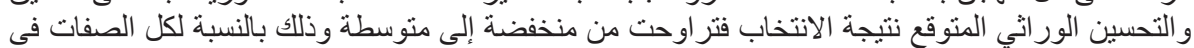

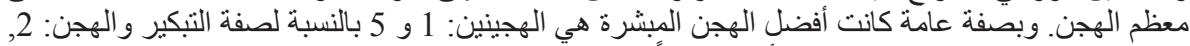

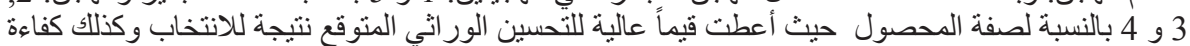

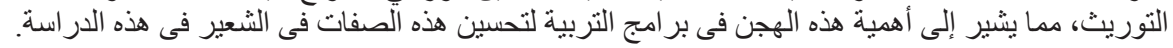

